\title{
Percepción y vulnerabilidad: los adolescentes en los territorios periféricos violentados
}

\author{
Alfonso Valenzuela-Aguilera \\ Universidad Autónoma del Estado de Morelos, Cuernavaca, México. \\ Email: aval@uaem.mx
}

\begin{abstract}
Resumen: En el presente trabajo revisamos el papel de la territorialidad como condición fundamental para la seguridad ciudadana desde la perspectiva de los jóvenes que habitan los territorios periféricos violentados en la Zona Metropolitana de Cuernavaca en Morelos, México. Para este efecto se diseñó la Primera Encuesta Sobre Percepción de la Inseguridad de los Alumnos de Bachilleratos Tecnológicos con el fin de detectar la percepción que los estudiantes tienen de la seguridad en dicha zona. Tomando como punto de partida la búsqueda de identidad de los adolescentes a partir del espacio geográfico como elemento articulador, identificamos, mediante la construcción de cinco indicadores, los mecanismos de apropiación del territorio como una estrategia para mantener el control social del espacio urbano.
\end{abstract} lencia.

Palabras clave: Percepción, vulnerabilidad, adolescentes, seguridad, vio-

\section{Perception and vulnerability: adolescents in disrupted peripheral territories}

\begin{abstract}
In this paper we assess the role that territoriality has as a fundamental condition for citizen security from the perspective of young people living in disrupted peripheral territories in the metropolitan area of Cuernavaca in Morelos, Mexico. For this purpose the First Survey on Perception of Insecurity in Technical High Schools, in order to detect the perception that students have on security issues, was designed. Taking as a point of departure the quest for identity in adolescents by means of the articulating features of geographical space, our research identifies, through the construction of five indicators, the mechanisms of appropriation of the territory as a strategy to maintain social control of urban space.
\end{abstract}

Keywords: Perception, vulnerability, adolescents, safety, violence.

\section{Percepção e vulnerabilidade: os adolescentes em territórios periféricos violentados}

Resumo: Neste artigo, revisamos o papel da territorialidade como condição fundamental para a segurança dos cidadãos a partir da perspectiva dos jovens que vivem em territórios periféricos violentadas na região metropolitana de Cuernavaca, em Morelos, México. Por este motivo desenhou-se a Primeira Pesquisa sobre Percepção da Insegurança entre alunos de Bacharelados Tecnológicos com a finalidade 
de detectar a percepção que os alunos têm da segurança nessaregião. Tomando como ponto de partida a busca da identidade dos adolescentes a partir do espaço geográfico como elemento articulador, identificamos, a través da construção de cinco indicadores, os mecanismos de apropriação do território como uma estratégia para manter o controle social do espaço urbano. violência.

Palavras-chave: Percepção, vulnerabilidade, adolescentes, segurança,

\section{La territorialidad entre los jóvenes y los mecanismos de eficacia colectiva}

La territorialidad se define como el patrón de comportamiento que reclama la apropiación y la defensa de zonas geográficas contra las amenazas de invasión de la propiedad (Becker, 1973; Sommer, 1969; Becker \& Mayo, 1971). En un trabajo pionero, Ardrey (1996) afirmaba que la territorialidad funciona como un comportamiento instintivo compartido con otros animales para defender un espacio frente a las invasiones. Sin embargo, la territorialidad va más allá de los instintos representando también un elemento fundamental para la creación de identidades a través de la configuración del entorno, en donde se producen experiencias comunes, encuentros y vínculos sociales. Por otra parte, Edney $(1972,1976)$ considera que la territorialidad representa un modo de abordar el control sobre el entorno social y, en el caso de las comunidades de alto riesgo, existen fuerzas que compiten por la apropiación y control del espacio, incluyendo grupos rivales, las fuerzas públicas y las organizaciones sociales, siempre que la dominación del territorio asegure la apropiación de lo que se considera valioso, estratégico o redituable (Altman, 1975).El territorio es fundamental para gestionar y organizar la vida cotidiana, ya sea de manera individual o grupal (Edney, 1976). No obstante, en entornos violentos el espacio es dividido, organizado y controlado por distintos actores que pueden participar en las esferas formales tanto como las informales. En entornos urbanos de alto riesgo el dominio territorial se ejerce incluso por medios ilegales con el fin de asegurar, proteger y dominar el espacio urbano, en el marco de una lucha de poder y control a largo plazo (Valenzuela y Monroy, 2014:1122).

En México la población más vulnerable a la violencia se encuentra entre los 20 y los 29 años (ENSAUT, 2012) y su territorialidad se expresa a través de la generación subjetiva de "mapas cognitivos" en donde se registran patrones de comportamiento que corresponden con "marcas" dentro del espacio urbano. Estos mapas se construyen con los códigos tanto antiguos como nuevos para darle sentido al territorio: parques se transforman en zonas de narcomenudeo, espacios que se convierten en fronteras que marcan lo que está más allá de los límites (off-limits), o la constante negociación por el uso del espacio público entre los habitantes (Low, 2000). Sin embargo, una función inexplorada de la territorialidad es su potencial para sostener la organización social, es decir, la manera como un 
grupo puede imponer su dominio a través del control contundente y la supremacía jerárquica. Tal dominación puede ser de una naturaleza compleja y contradictoria toda vez que las fuerzas legales, ilegales y paralegales pueden coexistir e interactuar en los distintos niveles en un mismo territorio.

Siguiendo el trabajo seminal de Janowitz (1991), el concepto de control social se puede definir como la capacidad de una organización social de regularse a sí misma de acuerdo con ciertos objetivos morales y colectivos. Sin embargo, esta capacidad puede ser de naturaleza legal o ilegal y seguir siendo relevantes para los distintos grupos sociales: por ejemplo, los jóvenes pueden ser responsables de algún tipo de organización social basado en principios y valores compartidos únicamente por sus miembros, además de que también pueden utilizar mecanismos de control social reconocidos en términos más amplios como "elementos irreductibles de coerción en un sistema legítimo de la autoridad” (Janowitz, 1991).

La territorialidad es una condición de primera importancia para la seguridad ciudadana, toda vez que el tipo de violencia sistémica que más daña a la población son los llamados delitos de alto impacto, los cuales tienen lugar en el territorio. Desde la psicología social se considera que "la territorialidad desempeña un rol importante en la vida del individuo, y está relacionada con cuestiones tales como la agresión interpersonal y el estatus social” (Holahan, 2010). En éste sentido, es importante destacar que los adolescentes se refieren al espacio geográfico como elemento articulador en la búsqueda de su identidad, integrando espacios como las calles, los parques, las plazas públicas y comerciales, en donde como individuos pueden dar evidencias de organización, poder y fuerza. Existen mecanismos de apropiación del territorio como parte de la identidad barrial que se basan en señales o marcas en el territorio (pintas, plaqueo, graffiti, tags, etc.) con lo que los jóvenes envían mensajes de dominio y apropiación sobre lugares estratégicos dentro de la Zona Metropolitana de Cuernavaca. Estos espacios constituyen elementos identitarios que no conviene transgredir cuando se trata de "territorios ocupados" por otros colectivos, de modo que los jóvenes se ven obligados a circular con un bajo perfil para no interferir con residentes de otras colonias, permitiéndoles así desplazarse sin generar enfrentamientos abiertos con grupos locales o con las fuerzas del orden.

La eficacia colectiva, entendida como la cohesión social entre los miembros de la comunidad aunada a su disposición para actuar en función del bien común, está directamente relacionada con los niveles de violencia. Partiendo de esta premisa intentaremos demostrar la relación existente entre los índices delictivos en comunidades específicas y la confianza mutua entre sus residentes. En sitios donde se concentra la pobreza, el desempleo y la inseguridad inmobiliaria, la eficacia colectiva llega a servir como mediador con relación a la violencia, lo que permitiría extender su potencial hacia asuntos de mayor relevancia para el bienestar de la comunidad como son la respuesta a recortes presupuestales en el sector social, la recolección de 
basura o el deterioro del entorno circundante. Dado que la respuesta comunitaria a la delincuencia es un atributo que permite activar las capacidades del trabajo en red, consideramos importante examinar el rol que la eficacia colectiva juega en la protección de los intereses comunes y esprecisamente la búsqueda del bienestar general a través de la seguridad social la que permite crear las condiciones mínimas para el desarrollo equilibrado de la sociedad.

\section{La percepción de la inseguridad en los territorios periféricos}

De acuerdo con el Censo de población y vivienda de 2010, el 56\% de la población del estado de Morelos presenta índices de marginación considerables (29\% medio, 23.5\% alto y 4\% muy alto), dentro de los cuales el $30 \%$ de los jóvenes entre 15 y 19 años presentan una condición de marginalidad (INEGI, II Conteo de población y vivienda 2005), lo cual los hace poco susceptibles a integrarse en la economía formal.

Siendo los adolescentes uno de los sectores de la población más vulnerables, se diseñó la Primera Encuesta Sobre Percepción de la Inseguridad de los Alumnos de Bachilleratos Tecnológicos en la Zona Metropolitana de Cuernavaca, Morelos 2010, la cual se enfocó a la percepción que los estudiantes de dichos centros educativos de nivel medio superior tienen sobre la seguridad urbana y quienes al momento de levantar dicha encuesta estaban inscritos en los semestres $1^{\circ}, 3^{\circ}$ y $5^{\circ}$ integrando una muestra de 941 encuestas levantadas en tres Centros de Estudios Tecnológicos Industrial y de Servicios (CETis) y un Centro de Bachillerato Tecnológico Industrial y de Servicios (CBTtis) de la Zona Metropolitana de Cuernavaca, seleccionados tanto por su ubicación como por las condiciones de vulnerabilidad por lo que respecta al tema de la inseguridad.

La encuesta tuvo como objetivo central el conocer los hábitos, las percepciones y el conocimiento que los adolescentes de las zonas periféricas de Cuernavaca tenían sobre las condiciones de inseguridad y la cultura ciudadana, recuperando la experiencia de la primera Encuesta Nacional Exclusión, Intolerancia y Violencia en Escuelas Públicas de Educación Media Superior, lo que permite comparar mecanismos de consulta similares por medio de encuestas al interior del universo que componen los jóvenes adolescentes de esa zona, que de acuerdo con el Censo General de Población y Vivienda del año 2010 sumaban un total de 74, 885 jóvenes entre 15 y 19 años. Con la intención de obtener un acercamiento al universo de los adolescentes de la ZMC, se calculó una muestra representativa al enfocar la investigación en aquellos quienes cursaban el bachillerato tecnológico. El proceso de selección se perfiló para éste tipo de instituciones de educación media superior, seleccionados por corresponder a zonas periféricas con altos índices delictivos y con niveles de marginalidad elevados. 


\section{Tabla 1.Población estudiantil base del ciclo escolar 2009-2010 de los Centros de Educación Tecnológica de la DGETI}

\begin{tabular}{|l|l|c|c|}
\hline No. de plantel & Plantel & Alumnos ciclo 2009-2010 & Muestra \\
\hline 43 & CETIS XOCHITEPEC & 2,100 & 229 \\
\hline 12 & CETIS VIVAC & 1,570 & 220 \\
\hline 166 & CBTIS TEJALPA & 1,500 & 289 \\
\hline 44 & CBTIS ALTAVISTA & 1,430 & 203 \\
\hline Total & & $\mathbf{6 , 6 0 0}$ & $\mathbf{9 4 1}$ \\
\hline
\end{tabular}

Fuente: Primera Encuesta Sobre Percepción de la Inseguridad de los Alumnos de Bachilleratos Tecnológicos en la Zona Metropolitana de Cuernavaca, Morelos 2010.

La encuesta incluyó cinco apartados temáticos, el primero de control y cuatro con secciones temáticas que abordan lo siguiente: a) Caracterización personal; b) Cultura ciudadana; c) Percepción de la inseguridad; y d) Estado de derecho y criminalización. El primer apartado asegura las fases de validación y procesos; la segunda identifica la posición y cohesión con la familia; el tercero explora el comportamiento en el espacio público de la ZMC así como la práctica diaria de su formación social; el cuarto evalúa la relación con el entorno urbano y detecta la llamada cifra negra del delito; la última parte refiere la percepción hacia las amenazas sobre la persona, la familia o la comunidad.

La encuesta tuvo como principal objetivo generar y concentrar información representativa acerca del grado de percepción de violencia en grupos de adolescentes que habitan las periferias de la ZMC. La información constituye un referente sobre las actividades, actitudes y sucesos que ocurren en el espacio inmediato, y si bien no se infiere que los resultados de la encuesta puedan generalizarse a todos los jóvenes que habitan la ZMC, los resultados sirven como referente tendencial e indicador de las actividades que se desarrollan en los polígonos de alto riesgo. La encuesta ayudó a dimensionar cualitativa y cuantitativamente la profundidad, conocimiento y percepción que tienen los adolescente sobre la seguridad personal y territorial en la ZMC, aportando elementos para explorar las emociones, los procesos de dominio territorial, la acción de la eficacia colectiva en cuanto a la posible solución o atenuación de la problemática de inseguridad, el grado de civilidad o de cultura ciudadana, la discriminación y victimización al interior de un grupo considerado como de alta vulnerabilidad.

El reactivo permitió detectar y caracterizar la prevalencia e incidencia del delito, así como los porcentajes de aquellos no denunciados y no registrados (cifra negra), la magnitud y características de la victimización, la percepción de los ciudadanos sobre la inseguridad, así como la percepción sobre la actuación de las autoridades ante las demandas de la ciudadanía. El estudio se centró en adolescentes urbanos alumnos de nivel medio supe- 
rior en bachilleratos tecnológicos en donde se aplicó un cuestionario autoadministrado seleccionándose un número de unidades (estudiantes) con el procedimiento de muestreo específico, equivalentes para propósitos de estimación, a una unidad seleccionada (estudiante) mediante un muestreo aleatorio simple. La información generada constituye una plataforma fundamental para comprender la percepción y posición que tienen los estudiantes de bachillerato ante el delito, la inseguridad y la violencia, pudiendo servir como punto de partida para el diseño de programas de apoyo para el adolescente que le permitan construir un proceso de crecimiento sostenido como persona, en la familia y como parte de una comunidad.

Los resultados preliminares de la encuesta se integran en cada una de las secciones, las cuales en un segundo momento servirán para la construcción de índices específicos ${ }^{1}$. En la primera sección se registró la información personal y se consideraron las preguntas que permitieron englobar la distribución de los alumnos encuestados de la muestra por plantel educativo, edad, sexo, principal actividad, estatus económico, lugar de procedencia, entre otros. La segunda sección identificó los valores comunitarios que sustentan la llamada eficacia colectiva, con el fin de explorar las actitudes y comportamientos del adolescente con respecto a sus derechos y deberes cívicos tanto individuales como colectivos, destacando sus redes de asociación y organización así como su actitud y comportamiento en el espacio urbano. De manera preliminar se registraron que tres de cada cuatro adolescentes consideran que las acciones conjuntas en la comunidad son prioritarias para el combate a la inseguridad, casi el 95\% refiere que el grupo de amigos juega un papel fundamental, mientras que solo un $41.1 \%$ considera importante la asociación comunitaria, dando con esto mayor prioridad a la acción que a la organización. La asociación territorial representaba un elemento importante toda vez que una tercera parte de los encuestados mantenía una relación estrecha con sus vecinos y solo un 3\% reportaba tener problemas con ellos, lo cual indica un cierto nivel de cohesión social en la colonia o comunidad. En ése sentido, los encuestados consideraban que para asegurar el control del espacio urbano era más importante la participación ciudadana (59.5\%), pero más aún lo era la actuación como grupo social organizado (91.6\%). La tercera sección explora los mecanismos informales del control social del espacio, en donde los adolescentes declararon su compromiso social: cerca de un $80 \%$ de ellos intervendría personalmente en presencia de actos vandálicos o criminales, mientras que solo un $20 \%$ se mantendría indiferente ante un evento de dicha naturaleza. Los estudiantes comprometidos (88.2\%), referían que la motivación y gratificación de realizar dicha intervención sería el simple hecho de haber actuado de manera correcta además de generar beneficios de carácter comunitario.

En la cuarta sección se registró la percepción de la inseguridad en el espacio urbano de la ZMC, la cual comprende desde la percepción sobre las zonas con mayor incidencia de delito en Cuernavaca, hasta la violencia percibida como usuarios del transporte público y en general del espacio urbano (calles, plazas, mercados, comercios, etc.). La percepción del miedo se manifestó de distintas maneras: un 78\% declaró que temían ser victimas 
de algún delito ya fuera al utilizar el transporte público o bien circulando en la vía pública, un $42 \%$ refirió un temor generalizado hacia a la delincuencia y un 30\% se sentía inseguro en su entorno inmediato, lo cual refleja las condiciones de peligrosidad que prevalecen en el entorno cotidiano. La problemática de la inseguridad no necesariamente se beneficia de un entorno degradado, como podría pensarse, ya que sólo un 7\% consideró que el descuido y la falta de mantenimiento era determinante para que un espacio fuera percibido como inseguro. En cambio un $25 \%$ consideraba necesario un número mayor de policías y patrullaje, y un 17\% refería la importancia de la iluminación pública. Sin embargo, es notable que aún así un 89\% de los encuestados percibía a Cuernavaca como un lugar seguro para vivir, descartando las zonas inseguras, las que un 64\% solía evitar en su vida cotidiana.

En la quinta sección se aborda la dimensión de género dentro de la percepción de la inseguridad, considerando que más de la mitad de las encuestas fueron contestadas por mujeres. Los bachilleres refieren actitudes más igualitarias con respecto a las cuestiones de género, en donde un 85.1\% de los encuestados coincide en la equidad de género y un $61.1 \%$ considera que la mujer resiente más los problemas de inseguridad. Asimismo, más de la mitad de los encuestados refiere que la mujer es agredida principalmente en la calle (51.9\%) y el $47.9 \%$ padece problemas de violencia intrafamiliar.En la sexta sección se aborda la victimización, en la denuncia por maltrato se refiere en apenas un $4 \%$, de los encuestados, aunque la posible razón de las pocas denuncias probablemente se refiera al $24 \%$ que desconfía de las autoridades. Si bien el tipo de violencia más recurrente es la verbal (52\%), uno de cada cuatro casos tiene que ver con violencia física, lo cual es alarmante al vincularse con la violencia de género.

En la última sección se exploró la convivencia en familia como vínculo tradicional, y como célula importante a la familia nuclear ${ }^{2}$; sin embargo nuevas configuraciones familiares están surgiendo compuestas mediante estructuras extensas, con actitudes y comportamientos que el adolescente refiere como elementos de cohesión familiar. De acuerdo con los resultados de la encuesta es la madre de la familia la que propicia dicha cohesión ya que un 52\% refiere la ausencia del padre en las reuniones familiares, afectando la integración de los hijos con los padres, y derivando en un marcado $39 \%$ de adolescentes que sienten temor hacia sus padres.

\section{La construcción de los índices de correlación estadística}

Para poder poder analizar y contrastar los elementos que conforman la percepción del miedo entre los bachilleres de la periferia de Cuernavaca, se construyeron seis índices con base a las respuestas proporcionadas por la población encuestada:

1. Índice de inseguridad

2. Índice de civilidad

3. Índice de territorialidad 
4. Índice de eficacia colectiva

5. Índice de estabilidad emocional

6. Índice de vulnerabilidad

Los índices se construyeron de acuerdo con los valores derivados de cada una de las preguntas del cuestionario, asignando para cada caso la suma y el peso relativo de las respuestas correspondientes, para calcular así los seis indicadores compuestos. Tomando como punto de partida que cada índice contiene un número determinado de variables que integran dimensiones específicas, el número de preguntas consideradas para cada índice son los siguientes: Percepción de Inseguridad (36); Civilidad (31); Territorialidad (27); Eficacia Colectiva (21); Estabilidad Emocional (22); y Vulnerabilidad (20). La suma de las respuestas se relativizó entre 100 para sacar los valores de aquellos índices que fueran comparables entre el $0.00 \mathrm{y}$ el 1.00, y cuyo resultado se almacenó en variables continuas, es decir con valores decimales entre el 0 y el 1.

Para efectos de análisis resumido, el tener valores continuos entre 0 y 1 puede llegar a considerarse como un rango muy amplio, dado que estos son valores continuos con decimales además de que se trata de seis índices. Por tanto, se categorizaron estos valores en rangos de 4, es decir, en variables categóricas nominales a los cuales se les denomina grados. Estos grados de estratificación se consideraron para los seis índices, con los siguientes valores:

- MUY ALTO: cuando el índice oscilaba entre 0.76 y 1.00

- ALTO: cuando el valor del índice estaba en el rango de 0.51 y 0.75

- MEDIO: cuando el valor que arroja el índice se encuentra entre 0.26 y 0.50

• BAJO: cuando el valor del índice se encuentra entre 0.00 y 0.25

Estos valores nominales aluden a una categorización uniforme para los seis índices, lo cual permite el hacerlos comparables entre ellos. Además realizaron pruebas de la relación que, de acuerdo con el comportamiento de los datos, pudieran confirmar la magnitud de correspondencia entre ellos. En otras palabras, mientras que los índices como tales sólo se le pueden aplicar medidas estadísticas de tendencia central, una vez categorizados, se les pueden aplicar medidas de dispersión. Asímismo, si bien los programas informáticos especializados como el SPSS ${ }^{3}$ permiten sacar dichas mediciones categorizando los valores numéricos, no es recomendable aplicar medidas de tendencia central como moda, mediana, y sobre todo media a los grados o categorización de índices por lo que ya se explicó en párrafos anteriores. De éste modo, la riqueza de categorizar las variables numéricas en grados y reducir las ocurrencias con el fin de globalizar en rangos, radica en la posibilidad de realizar otro tipo de pruebas estadísticas como las de chi-cuadrado y de correlación, con el objeto de observar la significatividad de los índices referidos de acuerdo con el tamaño de la muestra así como para encontrar las posibles relaciones entre cada uno de los seis índices categorizados en grados, partiendo del supuesto de que no existe relación alguna entre el comportamiento de los índices calculados. 


\section{Índice de percepción de Inseguridad}

Los resultados de la encuesta permitieron elaborar el índice de inseguridad para el que se tomaron en cuenta las preguntas que reflejan la percepción que los adolescentes tienen sobre la inseguridad en la ciudad. En cada una de las 36 preguntas el índice oscila entre 0 a 1, en donde un mayor acercamiento a 1 indica una mayor percepción de inseguridad en las calles y los espacios públicos. La agrupación de los índices formaron cuatro grados, como se indica a continuación:

Tabla 2. Índice y grado de percepción de inseguridad en la Zona Metropolitana de Cuernavaca, Morelos

\begin{tabular}{|l|l|l|}
\hline Índice & Grado & Porcentaje \\
\hline $0-0.25$ & Bajo & 4.5 \\
\hline $0.26-0.50$ & Medio & 78.8 \\
\hline $0.51-0.75$ & Alto & 16.6 \\
\hline $0.76-1.0$ & Muy Alto & 0.0 \\
\hline
\end{tabular}

Fuente: Primera Encuesta sobre Percepción de la Inseguridad en Bachilleratos

Tecnológicos de la Zona Metropolitana de Cuernavaca, Morelos 2010.

De acuerdo con éste índice tenemos que 5 de cada 100 alumnos percibe que la inseguridad es baja, un 79 por ciento consideraban que la inseguridad en la ciudad era media, mientras que cerca del 20 por ciento perciben que existe una grado de inseguridad alta en la ciudad de Cuernavaca.

Tabla 3. Grado de percepción de inseguridad

\begin{tabular}{|l|l|l|l|}
\hline Grado & Frecuencia & Porcentaje & \%Acumulado \\
\hline Muy alto & 0 & 0.0 & 0 \\
\hline Alto & 1098 & 16.6 & 16.6 \\
\hline Medio & 5202 & 78.8 & 95.5 \\
\hline Bajo & 300 & 4.5 & 100 \\
\hline Total & 6600 & 100 & \\
\hline
\end{tabular}

Fuente: Primera Encuesta sobre Percepción de la Inseguridad en Bachilleratos

Tecnológicos de la Zona Metropolitana de Cuernavaca, Morelos 2010. 
Polis, Revista Latinoamericana, Volumen 15, $N^{\circ}$ 44, 2016

\section{Índice de Civilidad (valores ciudadanos)}

La construcción de este índice recoge 31 preguntas en distintas secciones del reactivo, registrando opiniones sobre la percepción social acerca de personas indigentes, niños en situación de calle, vendedores ambulantes y los derechos humanos, entre otros. Se presenta a continuación el cuadro con los resultados con los grados de tolerancia con que se clasificaron, bajo, medio, alto y muy alto.

\section{Tabla 4. Índice y Grado de Civilidad en la Zona Metropolitana de Cuernavaca, Morelos}

\begin{tabular}{|l|l|l|}
\hline Índice & Grado & Porcentajes \\
\hline $0-0.25$ & Bajo & 9 \\
\hline $0.26-0.50$ & Medio & 36.8 \\
\hline $0.51-0.75$ & Alto & 62.3 \\
\hline $0.76-1.0$ & Muy Alto & 0.0 \\
\hline
\end{tabular}

Fuente: Primera Encuesta sobre Percepción de la Inseguridad en Bachilleratos

Tecnológicos de la Zona Metropolitana de Cuernavaca, Morelos 2010.

Tomando en cuenta los porcentajes de respuesta de los alumnos, es relevante destacar que el grado de civilidad está en los promedios altos, ya que 62 de cada 100 considera tener un alto grado de tolerancia, mientras que solo 9 de cada 100 tiene un grado bajo de tolerancia en su realidad cotidiana.

Tabla 5. Grado de Civilidad

\begin{tabular}{|l|l|l|l|}
\hline Grado & Frecuencia & Porcentaje & \% Acumulado \\
\hline Muy Alto & 0 & 0.0 & 0 \\
\hline Alto & 2428 & 62.3 & 36.8 \\
\hline Medio & 4110 & 36.8 & 62.3 \\
\hline Bajo & 62 & 0.9 & 100 \\
\hline Total & 6600 & 100 & \\
\hline
\end{tabular}

Fuente: Primera Encuesta sobre Percepción de la Inseguridad en Bachilleratos Tecnológicos de la Zona Metropolitana de Cuernavaca, Morelos 2010. 


\section{Índice de Territorialidad}

En la construcción de este índice se tomaron en cuenta 27 preguntas, entre las que destacan las relativas al uso de medios de transporte; los lugares de reunión así como su interacción con el espacio público (realizar acciones de apropiación como pintas, placas, mensajes); la percepción de pandillas en el espacio barrial; los cambios en la rutina cotidiana para disminuir las probabilidades de ser asaltado; la percepción sobre las colonias más inseguras; el grado de pertenencia y de territorialidad; así como la defensa o reconocimiento del espacio cotidiano.

\section{Tabla 6. Índice y Grado de Territorialidad en la Zona Metropolitana de Cuernavaca, Morelos}

\begin{tabular}{|l|l|l|}
\hline Índice & Grado & Porcentajes \\
\hline $0-0.25$ & Bajo & 1.5 \\
\hline $0.26-0.50$ & Medio & 85.3 \\
\hline $0.51-0.75$ & Alto & 13.1 \\
\hline $0.76-1.0$ & Muy Alto & 0.0 \\
\hline
\end{tabular}

Fuente: Primera Encuesta sobre Percepción de la Inseguridad en Bachilleratos

Tecnológicos de la Zona Metropolitana de Cuernavaca, Morelos 2010.

Un 85.3\% de las respuestas indica la importancia de territorialidad en la vida cotidiana y como elemento de pertenencia a la ciudad, a partir de lo que se infiere que es probable que el temor a represalias o a reacciones encontradas inhiba las acciones de apropiación espacial por parte de los adolescentes que deriven en una mayor seguridad espacial.

\section{Tabla 7. Grado de Territorialidad}

\begin{tabular}{|l|l|l|l|}
\hline Grado & Frecuencia & Porcentaje & \% Acumulado \\
\hline Muy alto & 0 & 0.0 & 0 \\
\hline Alto & 868 & 1.5 & 36.8 \\
\hline Medio & 5630 & 85.3 & 62.3 \\
\hline Bajo & 102 & 0.9 & 100 \\
\hline Total & 6600 & 100 & \\
\hline
\end{tabular}

Fuente: Primera Encuesta sobre Percepción de la Inseguridad en Bachilleratos

Tecnológicos de la Zona Metropolitana de Cuernavaca, Morelos 2010. 


\section{Índice de Eficacia Colectiva}

Este índice se trabajó con 21 preguntas y sus variables, las cuales reflejan respuestas positivas en torno a la convivencia y la participación de hombres y mujeres para mejorar las condiciones materiales de sus vecindarios, así como la cooperación moral para solucionar problemas de carácter social y cultural.

\section{Tabla 8. Índice y Grado de Eficacia Colectiva en la Zona Metropolitana de Cuernavaca, Morelos}

\begin{tabular}{|l|l|l|}
\hline Índice & Grado & Porcentajes \\
\hline $0-0.25$ & Bajo & 1.5 \\
\hline $0.26-0.50$ & Medio & 40.9 \\
\hline $0.51-0.75$ & Alto & 56.1 \\
\hline $0.76-1.0$ & Muy Alto & 0.0 \\
\hline
\end{tabular}

Fuente: Primera Encuesta sobre Percepción de la Inseguridad en Bachilleratos Tecnológicos de la Zona Metropolitana de Cuernavaca, Morelos 2010.

Para perfilar la Eficacia Colectiva se tomaron en cuenta las relaciones interpersonales con los vecinos; las prioridades de la ciudad desde una óptica ciudadana; y la asertividad ante la inseguridad en la ciudad, entre otros. Este índice se construyó con 13 preguntas de donde se desprenden 21 variables que reflejan la participación de los adolescentes en la organización y mantenimientos de espacios públicos seguros, así como la disponibilidad por coadyuvar con los vecinos para resolver problemas de seguridad, como un elemento de identidad y pertenencia al entorno comunitario.

Tabla 9. Grado de Eficacia Colectiva

\begin{tabular}{|l|l|l|l|}
\hline Grado & Frecuencia & Porcentaje & \% Acumulado \\
\hline Muy Alto & 98 & 15 & 15 \\
\hline Alto & 3701 & 56.1 & 57.6 \\
\hline Medio & 2699 & 40.9 & 98.5 \\
\hline Bajo & 102 & 1.5 & 100 \\
\hline Total & 6600 & 100 & \\
\hline
\end{tabular}

Fuente: Primera Encuesta sobre Percepción de la Inseguridad en Bachilleratos Tecnológicos de la Zona Metropolitana de Cuernavaca, Morelos 2010. 
El hecho que se se registren niveles de medio hacia arriba indica que el tejido social está relativamente cohesionado para resolver problemas de interés común. A partir de la percepción de los alumnos existe la disposición declarada por mantener el control de su entorno o territorio cotidiano, ya sea al interior de su escuela o en conjunto con los vecinos del vecindario.

\section{Índice de Estabilidad Emocional}

En la construcción del índice se utilizaron 22 preguntas que reflejan la capacidad emocional para hacer frente a un entorno que presenta factores de riesgo, señales de deterioro en el entorno, la sensación de temor o miedo así como el papel que juega la familia, la amistad, el amor o las instituciones educativas.

\section{Tabla 10. Índice y Grado de Estabilidad Emocional en la Zona Metropolitana de Cuernavaca, Morelos}

\begin{tabular}{|l|l|l|}
\hline Índice & Grado & Porcentajes \\
\hline $0-0.25$ & Bajo & 0.1 \\
\hline $0.26-0.50$ & Medio & 29.9 \\
\hline $0.51-0.75$ & Alto & 68.3 \\
\hline $0.76-1.0$ & Muy Alto & 1.7 \\
\hline
\end{tabular}

Fuente: Primera Encuesta sobre Percepción de la Inseguridad en Bachilleratos Tecnológicos de la Zona Metropolitana de Cuernavaca, Morelos 2010.

En la construcción de éste índice las preguntas refieren las locaciones de interacción social y de esparcimiento; las actividades que generan emociones fuertes; la respuesta a la frustración y la relación con los amigos, adultos y figuras de autoridad. 
Polis, Revista Latinoamericana, Volumen 15, $N^{\circ}$ 44, 2016

\section{Tabla 11. Grado de Estabilidad Emocional}

\begin{tabular}{|l|l|l|l|}
\hline Grado & Frecuencia & Porcentaje & \% Acumulado \\
\hline Muy Alto & 111 & 1.7 & 15 \\
\hline Alto & 4507 & 68.3 & 57.6 \\
\hline Medio & 1973 & 29.9 & 98.5 \\
\hline Bajo & 9 & 0.1 & 100 \\
\hline Total & 6600 & 100 & \\
\hline
\end{tabular}

Fuente: Primera Encuesta sobre Percepción de la Inseguridad en Bachilleratos Tecnológicos de la Zona Metropolitana de Cuernavaca, Morelos 2010.

Los bachilleres refieren frecuencias altas (68.3\%) y muy altas (1.7\%), lo que refleja la magnitud de los temores y los miedos que provienen de los factores de riesgo y que inciden en la alta deserción escolar, que para el ciclo escolar 2008-2009 en la Educación Media Superior fue del 15.9\%, equivalente a un estudiante abandonando sus estudios de Educación Media Superior en México cada 25 segundos. ${ }^{4}$

\section{Índice de Vulnerabilidad}

Para la construcción del índice de vulnerabilidad se utilizaron 20 preguntas y sus respectivas variables, las cuales reflejan la percepción de las acciones que los demás realizan en la vía publica, considerando como factor de riesgo el utilizar la calle para juegos, la capacidad de afrontar con eficacia los momentos de frustración, el percibir como segura el propio barrio o colonia, o la identificación de los barrios no seguros para poder evitar el tránsito en ellos. El índice de vulnerabilidad se ubica en un registro medio, lo que les permita vivir y convivir en armonía.

\section{Tabla 12. Índice y Grado de Vulnerabilidad en la Zona Metropolitana de Cuernavaca, Morelos}

\begin{tabular}{|l|l|l|}
\hline Índice & Grado & Porcentajes \\
\hline $0-0.25$ & Bajo & 28.8 \\
\hline $0.26-0.50$ & Medio & 70.1 \\
\hline $0.51-0.75$ & Alto & 3.1 \\
\hline $0.76-1.0$ & Muy Alto & 0.0 \\
\hline
\end{tabular}

Fuente: Primera Encuesta sobre Percepción de la Inseguridad en Bachilleratos

Tecnológicos de la Zona Metropolitana de Cuernavaca, Morelos 2010. 
El índice de vulnerabilidad se calculó a partir de las preguntas sobre el tipo de espacios públicos que se frecuentan; el deterioro del entorno como detonante de la inseguridad; y la percepción espacial de la inseguridad entre otros. Es consistente en tres cuartas partes de la población encuestada que existe un grado de vulnerabilidad medio con el hecho de habitar en la Zona Metropolitana de Cuernavaca.

Tabla 13. Grado de Vulnerabilidad

\begin{tabular}{|l|l|l|l|}
\hline Grado & Frecuencia & Porcentaje & \% Acumulado \\
\hline Muy Alto & 0 & 0.0 & 0.0 \\
\hline Alto & 206 & 3.1 & 3.1 \\
\hline Medio & 4625 & 70.1 & 73.2 \\
\hline Bajo & 1769 & 26.8 & 100 \\
\hline Total & 6600 & 100 & \\
\hline
\end{tabular}

Fuente: Primera Encuesta sobre Percepción de la Inseguridad en Bachilleratos

Tecnológicos de la Zona Metropolitana de Cuernavaca, Morelos 2010.

Análisis de las correlaciones entre los índices como factores que inciden en la inseguridad

El observar y determinar el comportamiento por medio de los índices de manera aislada no permite una lectura del comportamiento integral del fenómeno estudiado, haciéndose necesaria la interacción entre ellos de manera correlacional. Esto permite obtener un modelo que refleje la interacción entre causas y efectos con el fin de identificar los factores centrales que lo conforman pero tomando el índice de percepción de inseguridad como eje principal de las correlaciones con los otros cinco índices: civilidad, territorialidad, eficacia colectiva, estabilidad emocional y vulnerabilidad.

En otras palabras, sólo se le pueden aplicar medidas estadísticas de tendencia central a los índices como tales y es hasta que se categorizan que se les pueden aplicar medidas de dispersión. Para los seis índices calculados y sus respectivas agrupaciones en grados o categorías se incluyen 6600 casos, es decir, el total de la población estudiantil del ciclo 2009-2010, estimada mediante la muestra de encuestas levantadas en los cuatro Centros de Educación de Bachillerato Tecnológico de la Zona Metropolitana de Cuernavaca. Si se observa con detenimiento se concluye que en la totalidad de la muestra no se percibe una inseguridad muy alta, mientras que el 26.8\% de los alumnos considera tener un grado de vulnerabilidad bajo, pese a la inseguridad que se está viviendo en la región y a la incidencia de los medios de comunicación, lo que explica que un $68.3 \%$ registre un grado de estabilidad emocional alto. 


\section{Tabla 14. Grados de Inseguridad, Eficacia Colectiva, Civilidad, Estabilidad Emocional,}

\section{Territorialidad y Vulnerabilidad}

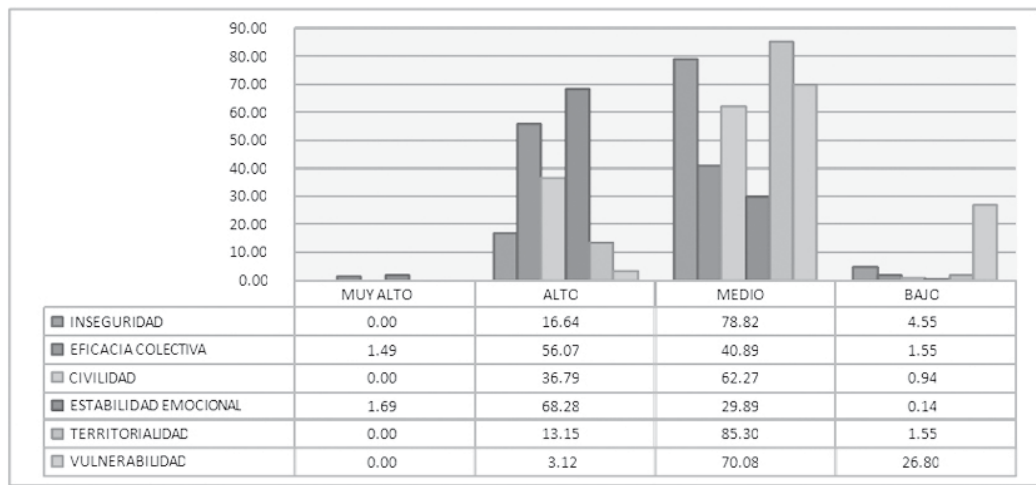

Fuente: Primera Encuesta sobre Percepción de la Inseguridad en Bachilleratos

Tecnológicos de la Zona Metropolitana de Cuernavaca, Morelos 2010.

El porcentaje más alto (85.30\%), corresponde a la percepción territorial media, lo cual podría interpretarse como una débil apropiación del espacio debido a que la inseguridad que se vive en la ZMC los aleja de identificarse espacialmente y generar un sentimiento de territorialidad. En éste sentido, la intención de categorizar las variables numéricas en grados y reducir las ocurrencias para globalizar en rangos, ayuda para obtener otro tipo de pruebas estadísticas como las pruebas de chi-cuadrada y de correlación, observándose la significatividad de los índices con respecto al tamaño de la muestra y para encontrar las posibles relaciones entre dichos índices, partiendo del supuesto de que no existe relación alguna entre el comportamiento de los índices calculados.Por tanto, se puede inferir del análisis de los comportamientos y tendencias algunas conclusiones incipientes, si bien no sean del todo suficientes para determinar de manera categórica las relaciones particulares entre los índices, por lo cual utilizamos las pruebas de chi cuadrada r de Pearson y derivándose las correlaciones que se presentan a continuación.

\section{Correlación entre Percepción de Inseguridad y Civilidad}

Si bien el conocer el comportamiento de las frecuencias de percepción de la población acusa una cierta relación de similitud, no sucede lo mismo con los índices como tales. Esto se debe a que mientras la inseguridad se relaciona con la falta de un orden urbano, legal y social, la eficacia colectiva, la civilidad, la estabilidad emocional, la territorialidad y la vulne- 
rabilidad son interpretaciones internas que los encuestados refieren a partir de su percepción de la inseguridad.

Tabla 15a. Grado de Inseguridad ^ ^ Grado de Civilidad

\begin{tabular}{|c|c|c|c|c|c|}
\hline \multicolumn{2}{|c|}{ Grado de Inseguridad } & \multicolumn{3}{|c|}{ Grado de Civilidad } & \multirow[t]{2}{*}{ Total } \\
\hline & & Alto & Medio & Bajo & \\
\hline \multirow[t]{2}{*}{ Muy Alto } & Recuento & 0 & 0 & 0 & 0 \\
\hline & Frecuencia esperada & & & & \\
\hline \multirow[t]{2}{*}{ Alto } & Recuento & 359 & 719 & 19 & 1097 \\
\hline & Frecuencia esperada & 403.5 & 683.2 & 10.3 & 1097 \\
\hline \multirow[t]{2}{*}{ Medio } & Recuento & 1988 & 3180 & 34 & 5202 \\
\hline & Frecuencia esperada & 1913.2 & 3239.9 & 48.9 & 5202 \\
\hline \multirow[t]{2}{*}{ Bajo } & Recuento & 80 & 211 & 9 & 300 \\
\hline & Frecuencia esperada & 110.3 & 186.8 & 2.8 & 300 \\
\hline \multirow[t]{2}{*}{ Total } & Recuento & 2427 & 4110 & 62 & 6599 \\
\hline & Frecuencia esperada & 2427 & 4100 & 62 & 6599 \\
\hline
\end{tabular}

Fuente: Elaboración propia con base en la Primera Encuesta sobre Percepción de la Inseguridad en Bachilleratos Tecnológicos de la Zona Metropolitana de Cuernavaca, Morelos 2010.

Tabla 15b. Pruebas de Chi Cuadrado Inseguridad y Civilidad

\begin{tabular}{|lrrrr|}
\hline \multicolumn{1}{|c}{ Pruebas de Chi Cuadrado } & \multicolumn{1}{c|}{ Valor } & Gl & Sig. Asintótica Bilateral \\
Chi-cuadrado de Pearson & $47.681^{\mathrm{a}}$ & 4 & .000 \\
Razón de verosimilitudes & 42.621 & 4 & .000 \\
Asociación lineal por lineal & 0.855 & 1 & .355 \\
No. de casos válidos & 6599 & & \\
\hline
\end{tabular}

A. 1 casillas (11.1\%) tienen una frecuencia esperada inferior a 5. La frecuencia mínima esperada es de 2,82.

Fuente: Elaboración propia con base en la Primera Encuesta sobre Percepción de la Inseguridad en Bachilleratos Tecnológicos de la Zona Metropolitana de Cuernavaca, Morelos 2010. 
Con respecto a la relación que guarda la inseguridad y la civilidad, las pruebas de Chi cuadrado $r$ de Pearson refieren un valor de 47.681, lo que indica que solo un $11.1 \%$ tuvo una frecuencia esperada menor a 5 de acuerdo con el modelo teórico y no obstante, la frecuencia mínima esperada dentro de los cálculos del modelo en SPSS fue de 2.82, un valor aún más bajo que el sugerido por dicho modelo. Si consideramos cuatro grados de libertad con un nivel de significancia para ambos extremos de .000 y estos valores se comparan con los valores teóricos del modelo de 0.05 , se rechaza la hipótesis nula que establece que no hay relación entre la inseguridad y la civilidad. Por consiguiente, se concluye que existe una relación directa entre la inseguridad y la civilidad, y esta mantiene una significancia asintótica bilateral concluyente en la relación de los dos índices. Los cuatro grados de libertad disminuyen debido a que la inseguridad no tiene frecuencias que indiquen que la población de alumnos encuestados crean que en la región de la ZMC existe una inseguridad muy alta, y de igual manera, tampoco perciben un alto grado de civilidad en la región de estudio o en el entorno de la población estudiantil encuestada.

La razón de verosimilitud, resultó ser significativa con un valor bilateral en las asíntotas de 0.000 ya que es menor a 0.05 , con lo cual se puede aceptar que civilidad si mejora o contribuye considerablemente a mejorar la inseguridad y debe considerarse como factor importante en relación con la inseguridad de la Zona Metropolitana de Cuernavaca. Por otra parte, la asociación lineal por lineal no resultó significativa ya que es mayor a 0.05 , el cual es el valor teórico de referencia del modelo, de modo que dicha asociación no revela una correlación alta (.855), es decir, que la prueba solo muestra una asociación significativa entre la inseguridad y la civilidad, pero no demuestra si mientras una baja la otra sube o viceversa; o por el contrario, si a medida que la civilidad aumenta la inseguridad baja, pero esto se demuestra con la última prueba que se mostrará más adelante. 
Tabla 16a. Grado de Inseguridad ^ $`$ Grado de Territorialidad

\begin{tabular}{|c|c|c|c|c|c|}
\hline \multicolumn{2}{|c|}{ Grado de Inseguridad } & \multicolumn{3}{|c|}{ Grado de Tenitorialidad } & \multirow[t]{2}{*}{ Total } \\
\hline & & Alto & Medio & Bajo & \\
\hline \multirow[t]{2}{*}{ Muy Alto } & Recuento & 0 & 0 & 0 & 0 \\
\hline & Frecuencia esperada & & & & \\
\hline \multirow[t]{2}{*}{ Alto } & Recuento & 194 & 886 & 18 & 1098 \\
\hline & Frecuencia esperada & 144.3 & 936.8 & 17.0 & 1098 \\
\hline \multirow[t]{2}{*}{ Medio } & Recuento & 664 & 4469 & 68 & 5201 \\
\hline & Frecuencia esperada & 683.3 & 4437.0 & 80.4 & 5201.0 \\
\hline \multirow[t]{2}{*}{ Bajo } & Recuento & 9 & 275 & 16 & 300 \\
\hline & Frecuencia esperada & 39.4 & 255.9 & 4.6 & 300 \\
\hline \multirow[t]{2}{*}{ Total } & Recuento & 867 & 5630 & 102 & 6599 \\
\hline & Frecuencia esperada & 867 & 5630 & 102 & 6599.0 \\
\hline
\end{tabular}

Fuente: Elaboración propia con base en la Primera Encuesta sobre Percepción de la Inseguridad en Bachilleratos Tecnológicos de la Zona Metropolitana de Cuernavaca, Morelos 2010.

Tabla 16b. Pruebas de Chi Cuadrado Inseguridad y Civilidad

\begin{tabular}{|lrcrl|}
\hline \multicolumn{1}{|c}{ Pruebas de Chi Cuadrado } & \multicolumn{1}{c|}{ Valor } & Gl & Sig. Asintótica Bilateral \\
Chi-cuadrado de Pearson & $75.380^{\mathrm{a}}$ & 4 & .000 \\
Razón de verosimilitudes & 73.656 & 4 & .000 \\
Asociación lineal por lineal & 47.156 & 1 & .000 \\
No. de casos válidos & 6599 & & \\
\hline
\end{tabular}

A. 1 casillas (11.1\%) tienen una frecuencia esperada inferior a 5 . La frecuencia mínima esperada es de 2,82.

Fuente: Elaboración propia con base en la Primera Encuesta sobre Percepción de la Inseguridad en Bachilleratos Tecnológicos de la Zona Metropolitana de Cuernavaca, Morelos 2010. 
La relación que guarda la inseguridad y la territorialidad de acuerdo con la prueba de Chi cuadrado r de Pearson refiere un valor de 75.380, lo cual indica que solo una casilla (11.1\%) tuvo una frecuencia esperada menor a 5 de acuerdo con el modelo teórico y no obstante, la frecuencia mínima esperada con los cálculos del modelo en SPSS fue de 4.64, también más baja que la sugerida por dicho modelo con 4 grados de libertad y con un nivel de significancia para ambos extremos de .000. Si estos números se comparan con los valores teóricos del modelo de 0.05 , se rechaza la hipótesis nula que establece que no hay relación entre la inseguridad y la territorialidad, con lo que se concluye que existe una relación directa entre la inseguridad y la territorialidad, con una significancia asintótica bilateral concluyente en la relación de los dos índices categorizados en grados. Los cuatro grados de libertad, bajan porque la inseguridad no tiene frecuencias que indiquen que la población de alumnos encuestados crea que en la región de la ZMC no exista una inseguridad muy alta, y de igual manera tampoco perciben un grado de territorialidad muy alto en el entorno de la población estudiantil encuestada. La razón de verosimilitud, resultó ser significativa con un valor bilateral en las asíntotas de 0.000 ya que es menor a 0.05 , con lo cual se puede aceptar que la civilidad mejora y contribuye considerablemente en el mejoramiento de la inseguridad y debe considerarse como factor importante en la formulación de políticas públicas para de la Zona Metropolitana de Cuernavaca. La asociación lineal por lineal resultó ser significativa ya que es menor a 0.05 e igual a 0.000 , por lo que dicha asociación refiere una cifra de 47.156, es decir que la prueba muestra revela una asociación significativa entre la inseguridad y la territorialidad.

Tabla 17a. Grado de Inseguridad ^ $\wedge$ Grado de Eficacia Colectiva

\begin{tabular}{|cc|rrrr|r|}
\hline \multirow{2}{*}{ Grado de Inseguridad } & \multicolumn{4}{c|}{ Grado de Eficacia Colectiva } & \multirow{2}{*}{ Total } \\
\cline { 3 - 5 } & Muy Alto & Alto & Medio & Bajo & \\
\hline Muy Alto & Recuento & 0 & 0 & 0 & 0 & 0 \\
& Frecuencia esperada & & & & & \\
\hline Alto & Recuento & 32 & 583 & 471 & 12 & 1098 \\
& Frecuencia esperada & 16.5 & 615.6 & 448.9 & 17.0 & 1098.0 \\
\hline Medio & Recuento & 67 & 3005 & 2050 & 81 & 5203 \\
& Frecuencia esperada & 78.0 & 2917.2 & 2127.4 & 80.4 & 5203.0 \\
\hline Bajo & Recuento & 0 & 113 & 178 & 9 & 300 \\
& Frecuencia esperada & 4.5 & 168.2 & 122.7 & 4.6 & 300.0 \\
\hline Total & Recuento & 99 & 3701 & 2699 & 102 & 6601 \\
& Frecuencia esperada & 99.9 & 3701.0 & 2699.0 & 102.0 & 6601.0 \\
\hline
\end{tabular}

Fuente: Elaboración propia con base en la Primera Encuesta sobre Percepción de la Inseguridad en Bachilleratos Tecnológicos de la Zona Metropolitana de Cuernavaca, Morelos 2010. 
Tabla 17b. Pruebas de Chi Cuadrado Eficacia Colectiva e Inseguridad

\begin{tabular}{|lrrrr|}
\hline \multicolumn{1}{|c}{ Pruebas De Chi Cuadrado } & Valor & Gl & Sig. Asintótica Bilateral \\
Chi-cuadrado de Pearson & $77.682^{\mathrm{a}}$ & 6 & .000 \\
Razón de verosimilitudes & 77.609 & 6 & .000 \\
Asociación lineal por lineal & 13.039 & 1 & .000 \\
No. de casos válidos & 6601 & & \\
\hline
\end{tabular}

A. 2 casillas (16.7\%) tienen una frecuencia esperada inferior a 5. La frecuencia mínima esperada es de 4,50.

Fuente: Elaboración propia con base en la Primera Encuesta sobre Percepción de la Inseguridad en Bachilleratos Tecnológicos de la Zona Metropolitana de Cuernavaca, Morelos 2010.

En éste caso la prueba de Chi cuadrado r de Pearson arrojó un valor de 77.628 , lo cual indica que tan solo un $16.7 \%$ tuvo una frecuencia esperada menor a 5 de acuerdo con el modelo teórico, y no obstante la mínima esperada con los cálculos del modelo en SPSS fue de 4.50, lo que quiere decir que resultó aún más baja que la sugerida por dicho modelo. Registrando 6 grados de libertad y con un nivel de significancia para ambos extremos de .000, estos valores pueden compararse con los valores teóricos del modelo de 0.05 , con lo que se rechaza la hipótesis nula que establece que no existe una relación entre la inseguridad y el eficacia colectiva, con lo que se concluye que existe una relación directa entre la inseguridad y la eficacia colectiva y tiene una significancia asintótica bilateral concluyente en la relación de los dos índices. Los grados de libertad se calculan con base al número de columnas y renglones generados a partir de la distribución y comportamiento de las frecuencias en ambos índices. En este caso, bajan porque el grado de inseguridad no tiene frecuencias que indiquen que la población de alumnos encuestados perciba que en la región de la Zona Metropolitana de Cuernavaca no existe una inseguridad muy alta, y en cambio perciben una muy alta eficacia colectiva en el entorno en el que la población estudiantil se desenvuelve.

La razón de verosimilitud, resultó ser significativa con un valor bilateral en las asíntotas de 0.000 ya que resultó menor a 0.05 , con lo cual se puede aceptar que la incorporación de la eficacia colectiva mejora sensiblemente a la inseguridad y debe considerarse como factor importante en relación con la inseguridad de la Zona Metropolitana de Cuernavaca. Asimismo, la asociación lineal por lineal resultó ser significativa al ser menor que 0.05 . No obstante, para el modelo en cuestión dicha asociación (13.039) no refleja mucho ya que son relativamente pocas variables, es decir, solo son dos las que se están contrastando. 
Polis, Revista Latinoamericana, Volumen 15, $N^{\circ}$ 44, 2016

Tabla 18a. Grado de Inseguridad ^ ^ Grado de Estabilidad Emocional

\begin{tabular}{|cc|rrrr|r|}
\hline \multirow{2}{*}{ Grado De Inseguridad } & \multicolumn{3}{|c|}{ Grado De Estabilidad Emocional } & \multirow{2}{*}{ Total } \\
\cline { 3 - 5 } & Muy Alto & Alto & Medio & Bajo & \\
\hline Muy Alto & Recuento & 0 & 0 & 0 & 0 & 0 \\
& Frecuencia esperada & & & & & \\
\hline Alto & Recuento & 12 & 756 & 330 & 0 & 1098 \\
& Frecuencia esperada & 18.5 & 749.7 & 328.3 & 1.5 & 1098.0 \\
\hline Medio & Recuento & 90 & 3536 & 1576 & 0 & 5202 \\
& Frecuencia esperada & 87.5 & 3552.1 & 67 & 7.1 & 5202.0 \\
\hline Bajo & Recuento & 9 & 214 & 67 & 9 & 299 \\
& Frecuencia esperada & 5.0 & 204.2 & 89.4 & 0.4 & 299.0 \\
\hline Total & Recuento & 111 & 4506 & 1973 & 9 & 6599 \\
& Frecuencia esperada & 111.0 & 4506.0 & $\mathbf{1 9 6 3 . 0}$ & 9.0 & 6599.0 \\
\hline
\end{tabular}

Fuente: Elaboración propia con base en la Primera Encuesta sobre Percepción de la Inseguridad en Bachilleratos Tecnológicos de la Zona Metropolitana de Cuernavaca, Morelos 2010.

Tabla 18b. Pruebas de Chi Cuadrado Eficacia Colectiva e Inseguridad

\begin{tabular}{|lrrrr|}
\hline \multicolumn{1}{|c}{ Pruebas de Chi Cuadrado } & \multicolumn{1}{c}{ Valor } & Gl & Sig. Asintótica Bilateral \\
Chi-cuadrado de Pearson & $201.598^{\mathrm{a}}$ & 6 & .000 \\
Razón de verosimilitudes & 67.913 & 6 & .000 \\
Asociación lineal por lineal & 0.661 & 1 & .416 \\
No. de casos válidos & 6599 & & \\
\hline
\end{tabular}

A. 2 casillas (16.7\%) tienen una frecuencia esperada inferior a 5. La frecuencia mínima esperada es de $\mathbf{. 4 1}$.

Fuente: Elaboración propia con base en la Primera Encuesta sobre Percepción de la Inseguridad en Bachilleratos Tecnológicos de la Zona Metropolitana de Cuernavaca, Morelos 2010.

En la prueba aplicada entre la inseguridad y la estabilidad emocional, el Chi cuadrado r de Pearson arrojó un valor de 201.598, lo que indica que en tan solo dos casillas, un $16.7 \%$ tuvo una frecuencia esperada menor a 5 de acuerdo con el modelo teórico, si bien la frecuencia mínima esperada con los cálculos del modelo en SPSS fue de .41, es decir, aún más baja que la sugerida por el modelo. Con 6 grados de libertad y con un nivel de 
significancia para ambos extremos de .000 . al compararse con los valores teóricos del modelo de 0.05 , se rechaza la hipótesis nula que establece que no hay relación entre la inseguridad y el estabilidad emocional, concluyéndose que existe una relación directa entre la inseguridad y la estabilidad emocional y tiene una significancia asintótica bilateral concluyente en la relación de los dos índices. Los 6 grados de libertad bajan porque el grado de inseguridad no tiene frecuencias que indiquen que la población de alumnos encuestados percibía que en la región de la Zona Metropolitana de Cuernavaca no hubiera una inseguridad muy alta, sino que por el contrario, los estudiantes encuestados se perciben con una muy alta estabilidad emocional.

La razón de verosimilitud, resultó ser significativa con un valor bilateral en las asíntotas de 0.000 ya que es menor a 0.05 , con lo cual se puede aceptar que la estabilidad emocional está relacionada con la inseguridad y debe considerarse como factor importante en relación con la inseguridad de la ZMC. La asociación lineal por lineal, resultó no ser significativa al ser mayor a 0.05 y al contrastar los dos índices y compararlos con el modelo se obtuvo un valor de 0.661 e cual revela una débil correlación entre ellos.

Tabla 19a. Grado de Inseguridad ^ ^ Grado de Vulnerabilidad

\begin{tabular}{|c|c|c|c|c|c|c|}
\hline \multicolumn{2}{|c|}{ Grado de Inseguridad } & \multicolumn{4}{|c|}{ Grado de Vulnerabilidad } & \multirow[t]{2}{*}{ Total } \\
\hline & & Muy Alto & Alto & Medio & Bajo & \\
\hline Muy Alto & $\begin{array}{l}\text { Recuento } \\
\text { Frecuencia esperada }\end{array}$ & 0 & 0 & 0 & 0 & 0 \\
\hline \multirow[t]{2}{*}{ Alto } & Recuento & 0 & 126 & 922 & 50 & 1098 \\
\hline & Frecuencia esperada & & 34.3 & 769.5 & 249.3 & 1098.0 \\
\hline \multirow[t]{2}{*}{ Medio } & Recuento & 0 & 80 & 3579 & 1544 & 5203 \\
\hline & Frecuencia esperada & & 162.4 & 3646.3 & 1394 & 5230.0 \\
\hline \multirow[t]{2}{*}{ Bajo } & Recuento & 0 & 0 & 125 & 175 & 300 \\
\hline & Frecuencia esperada & & 9.4 & 210.2 & 80.4 & 6601.0 \\
\hline \multirow[t]{2}{*}{ Total } & Recuento & 0 & 206 & 4626 & 1769 & 6601.0 \\
\hline & Frecuencia esperada & & & 4626.0 & 1769.0 & \\
\hline
\end{tabular}

Fuente: Elaboración propia con base en la Primera Encuesta sobre Percepción de la Inseguridad en Bachilleratos Tecnológicos de la Zona Metropolitana de Cuernavaca, Morelos 2010. 
Polis, Revista Latinoamericana, Volumen 15, $N^{\circ}$ 44, 2016

Tabla 19b. Pruebas de Chi Cuadrado Vulnerabilidad e Inseguridad

\begin{tabular}{|lrrrr|}
\hline \multicolumn{1}{|c}{ Pruebas De Chi Cuadrado } & Valor & Gl & Sig. Asintótica Bilateral \\
Chi-cuadrado de Pearson & $692.898^{\mathrm{a}}$ & 4 & .000 \\
Razón de verosimilitudes & 694.843 & 4 & .000 \\
Asociación lineal por lineal & 611.576 & 1 & .000 \\
No. de casos válidos & 6601 & & \\
\hline
\end{tabular}

A. 2 casillas $(0.0 \%)$ tienen una frecuencia esperada inferior a $5 . \mathrm{La}$ frecuencia mínima esperada es de 9.36 .

Fuente: Elaboración propia con base en la Primera Encuesta sobre Percepción de la Inseguridad en Bachilleratos Tecnológicos de la Zona Metropolitana de Cuernavaca,

Morelos 2010.

La relación que guarda la inseguridad y la vulnerabilidad de acuerdo con la prueba de Chi cuadrado r de Pearson, alcanzó un valor de 692.898, lo cual indica que la frecuencia menor a 5 esperada de acuerdo con el modelo teórico se presentó en cero casillas $(0 \%)$. No obstante que la frecuencia mínima esperada de acuerdo con los cálculos del modelo en SPSS era de 9.36, esta resultó aún más alta que la sugerida por el modelo. Con 4 grados de libertad y con un nivel de significancia para ambos extremos de .000, al compararse con los valores teóricos del modelo de 0.05 , se rechaza la hipótesis nula que establece que no existe una relación entre la inseguridad y la vulnerabilidad, concluyéndose que existe una relación directa entre la percepción de inseguridad y la vulnerabilidad, con una significancia asintótica bilateral concluyente en la relación de los dos índices categorizados en grados. Los 4 grados de libertad bajan porque la inseguridad no tiene frecuencias que indiquen que la población de alumnos encuestados consideraran que en la región de la Zona Metropolitana de Cuernavaca no hay una inseguridad muy alta, de igual manera que tampoco perciben muy alto grado de vulnerabilidad en la zona de estudio, o en el entorno espacial de la población estudiantil encuestada. Esta última conclusión, es muy significativa ya que la población estudiantil encuestada no se percibe como particularmente vulnerable, lo cual se deba quizá a la naturaleza misma de su juventud.

\section{Reflexiones finales: más allá de la percepción de inseguridad}

El presente trabajo sienta las bases para la construcción de un modelo que muestra y explica las causales objetivas de la percepción del adolescente con respecto a la inseguridad en el espacio, ubicando el problema dentro de un modelo teórico basado en la consideración de que son las diferencias e inequidades sociales y económicas localizadas en el territorio 
las que inciden de manera directa en la percepción de altos índices de inseguridad y violencia. Partiendo de una construcción teórica, se diseñó y aplicó la Primer Encuesta sobre Percepción de la Inseguridad de los Alumnos de Bachillerato Tecnológico en la Zona Metropolitana de Cuernavaca, con el fin de obtener información empírica que sustentara la percepción de inseguridad, combinando la reflexión racional, la observación de la realidad y la verificación correspondiente, de donde derivan los siguientes resultados:

\section{1) Percepción de inseguridad}

A partir de la información recabada en la encuesta y posterior tratamiento estadístico, se encontró que la percepción de inseguridad de la población objetivo se ubica dentro de una gradación media $(0.26-0.50)$ de 1.0. Es de todos modo relevante que un $78.8 \%$ de la población muestra no considere que la ZMC presenta riesgos y amenazas latentes como lo percibieron otros grupos de población en otras encuestas, lo cual sugiere que la población estudiantil de bachillerato se encuentra en una condición de resiliencia ${ }^{5}$, que probablemente le permitiría construir un proyecto de vida pese a las adversidades encontradas en su entorno inmediato.

La percepción de inseguridad moderada le permite al adolescente utilizar el transporte público en sus opciones de movilidad con ciertas reservas, así como utilizar espacios públicos, reunirse con sus pares o amigos para actividades de esparcimiento y socialización sin ser estigmatizados por su conducta por la familia e incluso la sociedad en su conjunto.

A partir de los resultados de la encuesta, destaca la necesidad de activar políticas de creación y renovación de espacios públicos que permitan a los usuarios disponer de opciones de diversión y convivencia sana, lo que redituaría en el beneficio, cohesión y fortalecimiento de la calidad de vida de los ciudadanos e incluso derivaría en la revalorización del territorio, disminuyendo con ello los factores de riesgo e inseguridad. En coincidencia con la primera Encuesta Nacional sobre Exclusión, Intolerancia en Escuelas públicas de Nivel Medio Superior (2008), los resultados de nuestro trabajo muestran que un $83.7 \%$ de los estudiantes de nivel medio superior se sienten vulnerados por la violencia, lo cual afecta las expectativas de vida, la movilidad cotidiana y la estabilidad emocional de dicho sector de la población. No obstante, los jóvenes contemplan su inserción en el mercado laboral al termino de sus estudios de bachillerato al contar con una preparación académica de nivel medio superior y una profesión de nivel técnico.

\section{2) Civilidad}

El índice de Civilidad se ubicó en un nivel medio (0.26 - 0.50), equivalente a un $62.3 \%$ de la muestra, para quienes consideran que la tolerancia y los valores ciudadanos en la ciudad son elementos indispensables para consolidar la civilidad mediante la observación de reglas formales e informales que permiten un trato cordial con los vecinos y reflejan una actitud de respeto hacia los espacios públicos al considerarlos como patrimonio de la 
sociedad y por tanto sensibles al marcaje con placas, mensajes o graffiti. Estas consideraciones deben ser tomadas en cuenta por las instituciones que promueven la adopción de valores entre la ciudadanía y en especial entre los adolescentes, esto con el objeto de restituir los principios universales, los derechos de los jóvenes y el respeto a las leyes.

\section{3) Territorialidad}

La territorialidad conlleva los mecanismos de apropiación, la construcción de un sentido de pertenencia y la defensa de un espacio particular. A partir de la aplicación de la encuesta se deriva que el 85.3\% de los adolescentes en la ZMC presentan un grado de territorialidad media $(0.26-0.50)$. El índice refiere también factores de movilidad intraurbana, es decir, que considera los espacios en donde se desplazan y trasladan dentro del territorio así como su traslado a las áreas de socialización y esparcimiento como parques, plazas públicas y comerciales, centros deportivos, y otros puntos de reunión. De dicho índice destaca la evidente falta de espacios públicos en donde desarrollar actividades recreativas, de esparcimiento y de socialización. No obstante, éste nivel medio de territorialidad no les permite a los adolescentes alcanzar una identificación espacial tal que contrarreste la percepción de violencia generalizada y en constante aumento. Aunado a esto, cuando la territorialidad emana de comunidades cerradas o fraccionamientos exclusivos, rompe con las funciones que favorecen la cohesión y el sentido comunitario, ya que si bien podría considerarse un proceso de apropiación del espacio, esta fomenta la fragmentación de la ciudad, alimentando los imaginarios de inseguridad en la ciudad. Es a partir de la encuesta que realizamos, destaca la importancia de la presencia del Estado en el territorio mediante el mantenimiento de condiciones de limpieza, iluminación y seguridad así como la provisión de las condiciones necesarias para promover la cohesión social, el sentimiento de pertenencia, y el mejoramiento de los niveles de confianza que aseguren un entorno amable para los ciudadanos.

\section{4) Eficacia colectiva}

Derivado de la encuesta y su procesamiento estadístico, la eficacia colectiva se ubicó dentro de un registro alto $(0.51-0.75)$, correspondiendo al 56.1\% de la población muestra, lo que refleja que existen mecanismos de interacción social internalizados entre los bachilleres quienes participan en la atención de los problemas de comunidad, inhibiendo con ello la formación de organizaciones dedicadas a actividades ilícitas y delictivas. En éste sentido, la eficacia colectiva busca proveer los medios e instrumentos (normas, estatutos) para la conformación de vínculos que aseguren los intereses de la comunidad.

\section{5) Estabilidad emocional}

A partir de la encuesta se registra una inestabilidad emocional alta $(0.51-0.75)$ y muy alta $(0.76-1.0)$ correspondiendo con un $68.3 \%$ y un $1.7 \%$ 
respectivamente, lo que refleja la capacidad de enfrentar los factores de riesgo que presentan en su entorno y también es signo de que se carece de factores de protección familiar a causa de la ausencia del padre (alcohólico, fumador, desempleado), violencia intrafamiliar, disolución familiar por abandono o divorcio, etc. En coincidencia con la primera Encuesta Nacional sobre Exclusión, Intolerancia en Escuelas públicas de Nivel Medio Superior (2008), los altos porcentajes de estrés y depresión que derivan en altas tasas de suicidio, es preocupante que el $46.6 \%$ de las alumnas mujeres a nivel nacional consideren que "han vivido situaciones en donde han deseado dejar de existir”, lo que demanda la localización temprana de alumnos con inestabilidad emocional, con el fin de instrumentar los programas de atención puntual para su recuperación.

Los retos de la inseguridad continúan siendo una cuestión de primer orden en América Latina y nuestra intención ha sido el identificar los parámetros bajo los cuales la sociedad civil puedaenfocarse para protegerse de la delincuencia, y sobre los cuales sea posible construir un modelo de intervención eficaz y anclado en la población residente interesada en mantener la cohesión social. Los altos índices de violencia e inseguridad de los últimos años han sido asociados al deterioro de las condiciones de vida de la población, destacando el nivel socioeconómico de los residentes, la inestabilidad laboral y residencial así como con la degradación del entorno físico y social. Sin embargo, los mecanismos estructurales que generan la pobreza tienen una estrecha relación con la localización de actividades criminales en ciertas áreas de la ciudad, y no obstante, el gasto público ha enfocado sus recursos en medidas que no han producido los efectos esperados: aumento en los sistemas de vigilancia, endurecimiento de condenas, compra de armamento o la contratación de mas elementos policíacos sin considerarlas relaciones territoriales de los ilícitos.

En el presente trabajo demostramos que la eficacia colectiva, entendida como la cohesión social entre los miembros de la comunidad aunada a su disposición para actuar en función del bien común, está directamente relacionada con los niveles de violencia. Por tanto, nuestro enfoque ha sido el de demostrar la relación existente entre los índices delictivos en comunidades específicas y la confianza mutua entre los residentes. La eficacia colectiva es un concepto generalizable a asuntos de mayor relevancia para el bienestar de la comunidad, como son la respuesta a recortes presupuestales en el sector social, la recolección de basura o el deterioro del entorno circundante. Dado que la respuesta individual a la delincuencia tiene una eficacia a nivel personal pero no permite activar las capacidades del trabajo en red, consideramos importante examinar el rol que la eficacia colectiva juega en la protección de los intereses colectivos.

El control social efectivo no es aquel de carácter oficial y obligatorio, sino del tipo informal y comunitario que busca el interés público. Dicho control refleja la capacidad de un grupo social para regular a sus miembros de acuerdo con principios deseables dirigidos a conseguir metas colectivas para el bienestar común. De este modo, el control social informal incluye el 
monitoreo espontáneo de grupos de juego infantil y juvenil, la disposición para intervenir en la prevención de actos delictivos, o la confrontación con personas que disturban o explotan el espacio público (como en le caso de la venta de estupefacientes, la prostitución o alcoholismo). Por tanto, la capacidad de los residentes para controlar procesos grupales y signos visibles de desorden social representa un mecanismo clave para influir en las oportunidades disponibles para actividades criminales en el vecindario.

La disposición de los residentes para intervenir en nombre del bien común va a depender en buena medida de la solidaridad y mutua confianza que exista entre los residentes. Es evidente que para que un residente intervenga para asegurar el bien común es necesario contar con reglas claras y construir una confianza básica entre los vecinos. Es importante destacar que tanto la eficacia colectiva como la individual están inmersas en contextos estructurales específicos, así como en una economía política mayor que estratifica a la población mediante características sociales precisas. Por tanto, superar la marcada estratificación socioeconómica que profundiza las desventajas concentradas en ciertas comunidades y que intensifica el aislamiento social de los menos favorecidos será fundamental para mantener y asegurar un control social efectivo del territorio. 


\section{Nota}

${ }^{1}$ La muestra fue de tipo polietápico aleatorio con un margen de error del $\%$, un nivel de confianza del $95 \%$ y el levantamiento de la encuesta fue auto-administrado en donde DEFT no fuera mayor a tres.

${ }^{2}$ Cerca del 75 por ciento de los hogares familiares son nucleares, es decir, aquellos en los que sus miembros mantienen una relación de parentesco consanguíneo o político de primer orden con el jefe del hogar. El resto son hogares con estructura extensa o ampliada, donde, reside un pariente del jefe de segundo grado o más. Boletín Nº195/10. México, D.F., 15 de mayo de 2010. Secretaria de Gobernación.Dirección General de Comunicación Social. Boletín de Prensa.

${ }^{3}$ Statistical Package for the Social Sciences Vers. 23.0

${ }^{4}$ Modelo Integral para la Atención y Acompañamiento en Educación Media Superior. Secretaria de Educación Media Superior. Octubre, 2010.

${ }^{5}$ La resilencia implica que las personas insertas dentro de una situación de adversidad, o expuestas a factores de riesgo, tienen la capacidad de transformar dichos factores para sobreponerse a la adversidad, crecer y desarrollarse adecuadamente, llegando a madurar como seres adultos competentes, pese a los pronósticos desfavorables. Programa Construye T, SEP, SEMS. 
Polis, Revista Latinoamericana, Volumen 15, No 44, 2016

\section{Bibliografía}

Alonso, Jorge (2007), “Política Social y Reforma democrática del Estado.”, en Ibero Forum Primavera, Tomo 3, Vol. 2.

Altman, Inwin(1975), The Environment and Social Behavior, Wadsworth, Belmont.

Ardrey, Robert (1966), The Territorial Imperative: A Personal Inquiry Into the Animal Origins of Property and Nations, Atheneum, Nueva York.

Becker, Franklin D. (1973), “Study of spatial markers”, en Journal of Personality and Social Psychology 26, 439-445.

Becker, Franklin D., \& Clara Mayo (1971), “Delineating personal distance and territoriality”, en Environment and Behavior, 12 (3), 375-381.

Edney, J. J. \& Jordan-Edney, N. L. (1974), “Territorial spacing on a beach”, en Sociometry, 37, 92-104.

Edney, J. J. (1976), “Human territories: comment on functional properties”, en Environment and Behavior, 8, 31-47.

ENSANUT (2012). Encuesta Nacional de Salud y Nutrición 2012, Instituto Nacional de Salud Pública. Ver http://ensanut.insp.mx/ informes.php\#.V4emYCPhDsc [consultado el 12 de mayo de 2016].

Holahan, Charles J. (2000),Psicología ambiental. Un enfoque general, Limusa, México.

Janowitz, Morris (1991), On Social Organization and Social Control, Chicago University Press, Chicago.

Ídem (1975), “Sociological theory and social control”, en American Journal of Sociology, 81:82-108, 1975.

Low, Setha M. (2000), On the Plaza: The Politics of Public Space and Culture, University of Texas Press, Austin.

Pastalan L. A. (1970), "Privacy as an expresion of human territoriality”, en Pastalan L.A. y D.H. Carson (eds.), Spatial behavior of older people, University of Michigan Press, Ann Harbor.

Sanchez-Jankowski, Martin (1991), Islands in the Street: Gangs and American Urban Society, University of California Press, Berkeley.

Sommer, Robert y Becker, Franklin D. (1969), “Territorial defense and the good neighbour”, en Joumal of Personality and Social Psychology 11, pp. 85-92. 
Sommer, Robert (1968), “Intimacy ratings in five countries”, en International Journal of Psychology 3, pp. 109-114.

Valenzuela Aguilera, Alfonso y Rafael Monroy-Ortiz (2014), “Formal/Informal/Ilegal: Los Tres Circuitos de la Economía Espacial en América Latina”, en Journal of Latin American Geography, Volume 13, Number 1, pp. 117-135.

Recibido: 11.07 .16

Aceptado: 30.07 .16 



\section{Propuestas y avances de investigación}

